\title{
Randomized Comparative Bioavailability of a Novel Three- Dimensional Printed Fast-Melt Formulation of Levetiracetam Following the Administration of a Single 1000-mg Dose to Healthy Human Volunteers Under Fasting and Fed Conditions
}

\author{
Sophie Boudriau ${ }^{1}$ (D) Cecilia Hanzel $^{1} \cdot$ Julie Massicotte $^{1} \cdot$ Laura Sayegh $^{1} \cdot$ \\ Jing Wang ${ }^{1}$ - Marc Lefebvre ${ }^{1}$
}

Published online: 30 March 2016

(c) The Author(s) 2016. This article is published with open access at Springerlink.com

\begin{abstract}
Background Rapidly disintegrating or 'fast-melt' oral formulations have been developed recently to facilitate drug intake among patients. Even though these formulations have helped to improve therapy adherence, some of their limitations include: the dissolution time, their facility to be swallowed, and the dosage strengths that may be accommodated. To overcome these limitations, a novel, porous, quickly disintegrating, and easier-to-swallow fastmelt formulation based on powder-liquid, three-dimensional printing (3DP) technology has been developed.

Objective To determine and compare the relative bioavailability of a novel 3DP fast melt containing levetiracetam in healthy male and female subjects.

Methods This study included 33 subjects in a three-way crossover design. The 3DP fast-melt formulation was compared against the conventional immediate-release tablet of levetiracetam $\left(\right.$ Keppra $\left.^{\circledR}\right)$ after a single 1000-mg dose administration under fasting conditions following the bioequivalence criteria used by the US Food and Drug Administration. This study also evaluated the food effect on the bioavailability of the levetiracetam 3DP fast melt. A small sip of liquid was used to administer the fast-melt formulation.

Results The novel 3DP fast melt showed rapid oral disintegration (mean duration of $11 \mathrm{~s}$ from a sip of water to
\end{abstract}

Sophie Boudriau

sboudriau@algopharm.com

1 Algorithme Pharma Inc., 575 Blvd. Armand-Frappier, Laval, QC H7V 4B3, Canada completion of swallowing) following its administration, and did not affect the pharmacokinetic profile of levetiracetam. A lower absorption peak was observed after administration of the 3DP fast melt under fed conditions, as expected. In addition, time of maximum measured plasma concentration was delayed by approximately $3.5 \mathrm{~h}$ under fed conditions. These effects are unlikely to be of clinical significance with long-term administration, and may help reduce the adverse events and facilitate compliance. Finally, no change in the oral mucosa was observed with the 3DP fast melt while being as safe and well tolerated as the standard levetiracetam tablet.

Conclusion This study quantified the rapid disintegration of the 3DP levetiracetam fast melt and confirmed its equivalent rate and extent of absorption to the conventional immediate-release tablet in the fasted state, using standard bioequivalence criteria.

\section{Key Points}

A novel levetiracetam three-dimensional printing (3DP) fast-melt formulation rapidly disintegrates within seconds in the mouth, when exposed to a small volume of liquid.

The levetiracetam 3DP fast melt was shown to be bioequivalent to the conventional tablet under fasting conditions.

The 3DP technology enables the production of highly porous, rapidly disintegrating oral formulations, which can incorporate higher doses of active drug, facilitating therapy adherence. 


\section{Introduction}

\subsection{Background and Objectives}

Levetiracetam is a second-generation antiepileptic drug (AED) currently approved by the US Food and Drug Administration (FDA) as an adjunctive treatment in patients with partial-onset seizures [1]. Levetiracetam is structurally unrelated to any other antiepileptic drug class and has a novel mechanism of action [1,2]. Levetiracetam is well tolerated, and adverse events (AEs) are limited primarily to the central nervous system such as asthenia, somnolence, dizziness, and headache [1,2].

Levetiracetam is rapidly and almost completely absorbed $(>95 \%)$ following oral administration and exhibits linear pharmacokinetics (PK) over a 500- to 5000-mg dose range. Levetiracetam is unlikely to compete with other drugs for protein-binding sites. Finally, its metabolism is minimal and not dependent on the hepatic cytochrome P450 enzyme system, indicating that clinical drug interactions are unlikely [1]. The efficacy and tolerability of levetiracetam make it a desirable AED [2]. Besides the oral tablet formulation, other formulations of levetiracetam include oral solution, extended-release tablets, and injection for intravenous use [3].

Poor adherence to therapy is an issue in the young, the older population, and in some other patients. Factors that may improve adherence include convenience of dosing, ease of use, drug tolerability, and patient satisfaction [4, 5]. Difficulty in swallowing is another barrier to medication adherence and is noted to occur in nearly one third of psychiatric patients, nearly a quarter of primary care patients, and more often in women than in men $[5,6]$.

The oral route remains the preferred route of drug administration owing to its convenience, good patient compliance, and low medicine production costs [7]. While an oral liquid (solution or suspension) supplied in bottles is easy to swallow, it lacks the convenience and portability of a premeasured unit dose, is not spill proof, and may not adequately mask the taste of active and inactive ingredients. 'Fast-melt' preparations such as orally disintegrating tablets (ODTs) were developed in the 1990s to address the swallowing difficulty of conventional tablets and capsules, without the limitations associated with oral liquids. Several ODT products are currently marketed for drugs in various therapeutic areas including AEDs [8,9]. While these ODT products have been designed to be administered without water and disintegrate on the tongue in generally less than $30 \mathrm{~s}$, the vast majority have only incorporated limited amounts of the active drug product (e.g., $10-30 \mathrm{mg}$ ), which has limited the application of ODT technology to higherdose drug products. A recent innovation, based on powder- liquid three-dimensional printing (3DP) technology, has been developed to overcome these limitations. The 3DP technology approach enables the production of highly porous, rapidly disintegrating oral formulations, which can incorporate up to $1000-\mathrm{mg}$ of the active drug product per tablet. Importantly, the 3DP technology approach incorporates various taste-masking techniques and requires each unit dose to be taken with a small volume of liquid for ease of administration and swallowing.

When comparing an ODT with a conventional tablet formulation, one of the criteria required by the regulatory authorities is to conduct a comparative bioavailability study, which for example may use bioequivalence criteria to confirm similar PK behavior despite the differences in form, mode of administration, and final formulation. Although different types of rapidly disintegrating tablets exist, with different rates of disintegration and/or dissolution, the bioequivalence criteria are still the same.

The primary objectives of the study were to determine and compare the levetiracetam plasma concentrations following a single 1000-mg administration of the levetiracetam 3DP fast-melt formulation (test product) and an immediate-release, film-coated Keppra ${ }^{\circledR}$ tablet (reference product) in normal healthy volunteers, and to evaluate the effect of food consumption on the PK profile of levetiracetam 3DP fast melt. The secondary objective of the study was to determine the safety and tolerability of levetiracetam 3DP fast melt compared with Keppra film-coated tablets in healthy volunteers. In addition, the disintegration time for the 3DP fast melt was measured.

\section{Methods}

\subsection{Study Design and Treatment}

This randomized, open-label, single-dose, three-period, three-treatment, three-sequence crossover study was conducted in October 2013 in Canada (Algorithme Pharma Inc., Montreal, Quebec, Canada). Thirty-three subjects were randomly assigned to one of three sequences: $\mathrm{ABC}$, $\mathrm{BCA}$, and $\mathrm{CAB}$. The randomization code list was generated using the Algorithme Pharma-developed application named Clinical according to the study design, the number of subjects, and the sequence of drug administration, and using blocks with a size of three. In each study period, subjects fasted overnight prior to and for at least $4 \mathrm{~h}$ after dosing or prior to receiving a FDA-compliant, high-fat, high-calorie meal. The treatment periods were separated by a washout period of 7 days. Subjects were dosed with the levetiracetam test product (Treatment-1, A) and the reference product (Treatment-2, B) under fasting conditions, 
and also received the test product $30 \mathrm{~min}$ after an FDAcompliant, high-fat, high-calorie breakfast (Treatment-3, C).

Following intra-oral placement of the levetiracetam 3DP fast melt (test product) directly on the tongue, subjects were instructed to wet their mouth by taking a sip from a cup containing $30 \mathrm{~mL}$ of water at ambient temperature without swallowing the dosage unit. Once the dosage unit was disintegrated, subjects were asked to swallow the medication. The elapsed time between water intake and completion of swallowing was recorded for all subjects. This elapsed time from sip to swallowing represented the recorded disintegration time for the fast-melt test product. Upon request by the subject, additional water (in amounts of $30 \mathrm{~mL}$ ) was to be provided; and actual amounts of water intake were documented. The reference product (Keppra ${ }^{\circledR}$ tablet) was administered with $240 \mathrm{~mL}$ of water at ambient temperature.

A novel, 1000-mg 3DP fast-melt formulation of levetiracetam manufactured by Aprecia Pharmaceuticals Company (Blue Ash, OH, USA), was compared with Keppra $^{\circledR}$ 1000-mg film-coated tablets, manufactured by UCB Inc., Smyrna, GA, USA. Study drugs were stored at the study site at a controlled room temperature $\left(15-30{ }^{\circ} \mathrm{C}\right)$.

\subsection{Participants}

Subjects were healthy men or non-pregnant women, aged between 18 and 50 years, with a body mass index $\geq 18.50$ and $<30.00 \mathrm{~kg} / \mathrm{m}^{2}$. Efforts were made to enroll a similar proportion of male and female subjects. Subjects were screened within 28 days prior to drug administration. The screening visit included medical history and demographic data collection including alcohol and smoking habits. A complete physical examination (head, eyes, ears, nose, throat, neck, chest, back, abdomen, extremities, and neurological function), the measurement of vital signs (pulse rate, blood pressure, and body temperature), a 12-lead electrocardiogram (ECG), neurological function (cognitive, cranial nerves, sensory, motor, coordination, and equilibrium), and laboratory tests (hematology, serum chemistry, and urinalysis) were performed.

No prescription medication, apart from hormonal contraceptives and hormone replacement therapy, was allowed for 28 days prior to dosing and during the study. Over-thecounter products had to be stopped 7 days before dosing, as well as grapefruit/pomelo nutritional sources. Only non- or ex-smokers were allowed, and alcohol and food or beverages containing xanthines were to be avoided $58 \mathrm{~h}$ before each dosing. Subjects were asked to refrain from heavy exercise during the study. No concomitant medications were allowed during the study.

\subsection{Pharmacokinetic Endpoints}

In each study period, blood samples were collected prior to and $0.17,0.25,0.33,0.50,0.67,0.83,1.00,1.33,1.67,2.00$, 2.50, 3.00, 3.50, 4.00, 5.00, 6.00, 8.00, 10.00, 12.00, 16.00, 24.00 , and $36.00 \mathrm{~h}$ after drug administration for PK measurements of levetiracetam.

Blood samples were collected in pre-cooled $\mathrm{K}_{2}$ EDTA Vacutainers. Following blood collection, samples were centrifuged at a temperature of $4{ }^{\circ} \mathrm{C}$ nominal and at approximately $1500 \mathrm{~g}$ for $10 \mathrm{~min}$. The plasma obtained was transferred in polypropylene culture tubes and was maintained in an ice-water bath until stored in the clinical freezers. Tubes were labeled with a code number that did not reveal the identity of the treatments. Samples were frozen at a temperature of $-20{ }^{\circ} \mathrm{C}$ nominal.

Levetiracetam plasma samples were assayed through a validated liquid chromatography-tandem mass spectrometry analytical method. Sample pre-treatment involved the protein precipitation extraction of levetiracetam from $0.1 \mathrm{~mL}$ of human plasma; levetiracetam-D6 was used as the internal standard. The compound was quantified using reversed-phase, high-performance liquid chromatography with a triple quadrupole mass spectrometer equipped with a turbo-assisted ionspray ionization source in positive mode over a theoretical concentration range of $0.250-65.000 \mu \mathrm{g} /$ $\mathrm{mL}$. The concentrations were calculated using peak area ratios and the linearity of the calibration curve was determined using least-squares regression analysis employing a weighted $\left(1 / x^{2}\right)$ linear $(y=m x+b)$ for levetiracetam.

The following PK parameters were calculated: maximum observed plasma concentration $\left(C_{\max }\right)$, time of maximum measured plasma concentration $\left(T_{\max }\right)$, cumulative area under the plasma concentration time curve calculated from 0 to time of the last quantifiable concentration using the trapezoidal rule (linear trapezoidal linear interpolation) $\left(\mathrm{AUC}_{0-\mathrm{T}}\right)$, extrapolated area under the plasma concentration time curve to infinity $\left(\mathrm{AUC}_{0-\infty}\right)$, and half-life of elimination $\left(T_{\text {half }}\right)$. The terminal-phase estimation was based on maximizing the coefficient of determination. Non-compartmental PK analyses were performed using Phoenix ${ }^{\circledR}$ WinNonlin ${ }^{\circledR}$, version 6.3 (Certara USA, Inc., Princeton, NJ).

\subsection{Safety and Tolerability Endpoints}

Safety monitoring during the study was performed through vital signs and 12-lead ECGs performed regularly after each drug administration. Neurological function tests were performed before the subject's departure from the study site. A complete physical examination including vital sign measurements, a 12-lead ECG, and laboratory testing was 
also performed at the end of the study. Subjects were regularly questioned on their health status during confinement, and AEs were recorded, symptoms thoroughly described, and followed up until satisfactory resolution.

Oral mucosal safety and local tolerability were assessed by monitoring erythema, edema, or other AEs. A visual inspection of the tongue, palate, and the oral mucosa was performed prior to and $0.5 \mathrm{~h}$ after drug administration. This inspection was also performed immediately after drug administration as part of treatment compliance. Throughout study confinement, the subjects were asked to self-report any signs or symptoms of mouth irritation. Observation of the tongue, palate, buccal mucosa, and surrounding area was documented by using a four-point severity scale $(0=$ no irritation, $1=$ mild irritation, $2=$ moderate irritation and $3=$ severe irritation). If tissue debris or sloughing was present on the surface mucosa, a description that included the degree (mild, moderate, or severe) was noted.

Subjects were asked to complete a questionnaire on their experience with the study products.

\subsection{Statistical Methodology}

The intra-subject variation following a single dose of levetiracetam was estimated to be less than $20 \%$. Given that the test-to-reference ratio of geometric LSmeans was expected to fall within 92.5 and $107.5 \%$, it was estimated that 33 subjects were needed to be included in the study to meet the 80.00-125.00\% confidence interval (CI) range with a statistical a priori power of at least $80 \%$.

Subjects who provided evaluable data for at least one of the comparisons of interest (Treatment-1 vs. Treatment-2 or Treatment-3 vs. Treatment-1) were included in the pharmacokinetic and statistical analysis. The natural logarithm (ln) of $C_{\text {max }}, \mathrm{AUC}_{0-\mathrm{T}}$, and $\mathrm{AUC}_{0-\infty}$, and the rank transformation of $T_{\max }$ were used for statistical inference. Statistical analyses were performed with an analysis of variance (ANOVA) model and the two-sided $90 \%$ CI of ratio of geometric LSmeans was obtained between both products under fasting conditions (Treatment-1 vs. Treatment-2). Food effect of levetiracetam 3DP fast melt was assessed using the ratio of geometric LSmeans with corresponding $90 \%$ CI calculated from the exponential of the difference between fed and fasting conditions (Treatment-3 vs. Treatment-1).

Statistical analyses were performed using SAS version 9 (SAS Institute, USA). SAS PROC MIXED was used for the ANOVA model. The fixed factors included in the model were the treatment received, the period at which it was given, as well as the sequence in which each treatment was received. A random factor was added for the subject effect (nested within sequence). Statistical significance was assessed at the two-sided $5 \%$ level.
The safety population included participants who received at least one treatment. Safety and tolerability endpoints were summarized using descriptive statistics.

\section{Results}

\subsection{Demographic}

The 33 subjects included in this study (Table 1) were healthy male and female individuals, most commonly White $(82 \%)$, aged between 18 and 48 years (safety population). All subjects in this study were non-smokers. The results of the screening urinary drug and serology tests ( $\beta$-human chorionic gonadotropin qualitative) indicated that all subjects were suitable for inclusion in the study. All subjects received at least one dose of the study drug and were included in the safety analysis and oral mucosa tolerability evaluation. Two subjects were withdrawn from the study before dosing of period 2 , one because of a positive cannabinoids test result and one owing to an unmet protocol requirement that could have had an impact on his PK profile for this period (critical breakfast not completed within $30 \mathrm{~min}$ before dosing). The latter returned to the clinical site for period 3 of the study. Thus, 32 subjects were included in the PK and statistical analysis (PK population).

The study subjects included in the PK population were fairly distributed between men and women, mean age of 31 years with a small percentage of subjects $(4 \%)$ aged older than 41 years; and the mean body mass index was $24.5 \mathrm{~kg} / \mathrm{m}^{2}$.

Table 1 Demographic and baseline characteristics of the subjects included in the study

\begin{tabular}{lc}
\hline Characteristic & $\begin{array}{c}\text { Overall (safety population) } \\
N=33\end{array}$ \\
\hline Mean age (range), years & $30(18-48)$ \\
Age groups, years, $n(\%)$ & $29(87.9)$ \\
$18-40$ & $4(12.1)$ \\
$41-48$ & \\
Sex, $n(\%)$ & $19(58)$ \\
Male & $14(42)$ \\
Female & \\
Race, $n(\%)$ & $27(82)$ \\
White & $4(12)$ \\
Black & $1(3)$ \\
Asian & $1(3)$ \\
Others & $24.35(19-29)$ \\
Mean BMI (range), $\mathrm{kg} / \mathrm{m}^{2}$ &
\end{tabular}

$B M I$ body mass index 


\subsection{Pharmacokinetics}

\subsubsection{DP Dosage Form and Water Intake}

The 3DP fast-melt formulation of levetiracetam is designed to disperse in the mouth within seconds in the presence of a sip of water for ease of administration and swallowing. The amount of water consumed by subjects during test product administration was measured (range $2-30 \mathrm{~mL}$, see Table 2), and the elapsed time between the sip of water and swallowing of the completely dispersed test product was recorded for each subject. Of those administered the levetiracetam 3DP fast-melt formulation, the mean amount of water consumed was consistent between the fasting and

Table 2 Total volume of water consumed by subjects

\begin{tabular}{lll}
\hline & Test fast & Test fed \\
\hline$N$ & 32 & 32 \\
Mean (SD) (mL) & $12(5)$ & $14(7)$ \\
Median (mL) & 12.5 & 13.0 \\
\hline
\end{tabular}

$S D$ standard deviation

Table 3 Elapsed time between the sip of water and swallowing of the medication

\begin{tabular}{lll}
\hline & Fasting & $\mathrm{Fed}^{\mathrm{a}}$ \\
\hline$N$ & 32 & 32 \\
Mean (SD) (s) & $11(11)^{\mathrm{b}}$ & $11(6)$ \\
Median (s) & 7.5 & 10.0 \\
\hline
\end{tabular}

$S D$ standard deviation

a The drug was administered $30 \mathrm{~min}$ after the start of the breakfast

${ }^{\mathrm{b}}$ One subject had an elapsed time of $62 \mathrm{~s}$ recorded with the initial administration. Upon subsequent exposure, the elapsed time from sip to swallowing the 3DP fast-melt formulation was reduced to $13 \mathrm{~s}$ for this subject fed conditions. Similar elapsed disintegration time was observed during fasting and fed conditions, with a mean overall time of $11 \mathrm{~s}$ (Table 3 ).

\subsection{Pharmacokinetics and Bioequivalence Assessment}

The mean PK values of levetiracetam after oral administration as a 3DP fast melt or as a conventional immediaterelease tablet are summarized in Table 4.

Under fasting conditions, the ranked time to peak concentrations for the 3DP fast melt (test) and the reference tablet were comparable $(p=0.5444)$, and both showed a very fast absorption within approximately $30 \mathrm{~min}$. Similar values were also evident for the main $\mathrm{PK}$ parameters evaluated in this study $\left(C_{\max }, \mathrm{AUC}_{0-t}\right.$, and $\left.\mathrm{AUC}_{0-\text { inf }}\right)$, and the terminal elimination half-life was approximately $7.1 \mathrm{~h}$ for both formulations.

The $90 \%$ CI for evaluating the bioequivalence and the corresponding point estimates of the relative bioavailability for the ln-transformed PK parameters are summarized in Table 5. The geometric LSmeans for $C_{\max }, \mathrm{AUC}_{0-\mathrm{t}}$, and $\mathrm{AUC}_{0-\text { inf }}$ were $106.45,102.94$, and $102.78 \%$, respectively. The results presented herein show that the criteria used to assess the relative bioavailability between these two formulations under fasting conditions were all contained entirely within the range of $0.80-1.25$.

The mean plasma concentration-time profile of levetiracetam 3DP tablet compared with that of the intact conventional tablet is presented in Fig. 1.

\subsection{Food Effect}

The levetiracetam 3DP fast melt was administered after a high-fat, high-calorie breakfast (136 kcal protein; $284 \mathrm{kcal}$ carbohydrate; $517.5 \mathrm{kcal}$ fat), which was expected to
Table 4 Comparison of the pharmacokinetic parameters: levetiracetam 3DP fast melt vs. conventional levetiracetam tablet

\begin{tabular}{|c|c|c|c|c|c|c|}
\hline \multirow[t]{2}{*}{ Parameter } & \multicolumn{2}{|c|}{$\begin{array}{l}\text { 3DP fast melt } \\
\text { Fasted } \\
N=32\end{array}$} & \multicolumn{2}{|c|}{$\begin{array}{l}\text { Conventional tablet } \\
\text { Fasted } \\
N=32\end{array}$} & \multicolumn{2}{|c|}{$\begin{array}{l}\text { 3DP fast melt } \\
\text { Fed } \\
N=31\end{array}$} \\
\hline & $\operatorname{Mean}^{\mathrm{a}}$ & (c.v.\%) & $\operatorname{Mean}^{\mathrm{a}}$ & (c.v.\%) & $\operatorname{Mean}^{\mathrm{a}}$ & (c.v.\%) \\
\hline$T_{\max }(\mathrm{h})$ & 0.58 & $(73.7)$ & 0.58 & $(69.9)$ & 4.00 & $(21.6)$ \\
\hline$C_{\max }(\mu \mathrm{g} / \mathrm{mL})$ & 33.273 & $(30.1)$ & 30.480 & $(19.0)$ & 20.481 & $(16.3)$ \\
\hline $\mathrm{AUC}_{0-\mathrm{T}}(\mu \mathrm{g} \cdot \mathrm{h} / \mathrm{mL})$ & 283.689 & $(20.0)$ & 274.934 & $(18.2)$ & 262.550 & $(15.1)$ \\
\hline $\operatorname{AUC}_{0-\infty}(\mu \mathrm{g} \cdot \mathrm{h} / \mathrm{mL})$ & 292.927 & (19.9) & 284.300 & $(18.0)$ & 272.565 & $(15.2)$ \\
\hline$T_{\text {half }}(\mathrm{h})$ & 7.13 & (13.3) & 7.14 & (16.3) & 7.19 & $(15.3)$ \\
\hline
\end{tabular}

$T_{\max }$ time to maximum observed plasma concentration, $C_{\max }$ maximum observed plasma concentration, $A U C_{0-T}$ area under the plasma concentration-time curve from time zero to the time of the last measurable concentration, $A U C_{0-\infty}$ area under the plasma concentration-time curve from time zero to infinity, $T_{\text {half }}$ terminal elimination half-life, c.v. coefficient of variation, $3 D P$ three-dimensional printing

${ }^{\text {a }}$ Median for $T_{\max }$ 
Table 5 Ninety percent confidence intervals of ln-transformed pharmacokinetic parameters of levetiracetam after a single 1000-mg oral dose of the 3DP fast-melt (test formulation) and the conventional tablet (reference formulation) under fasting conditions in healthy volunteers

\begin{tabular}{llcc}
\hline Parameter & Ratio (test/reference) & \multicolumn{2}{c}{$90 \%$ confidence intervals } \\
\cline { 3 - 4 } & & Lower & Upper \\
\hline $\ln C_{\text {max }}$ & 106.45 & 99.74 & 113.62 \\
$\operatorname{lnAUC}_{0-\mathrm{T}}$ & 102.94 & 100.93 & 105.00 \\
$\operatorname{lnAUC}_{0-\infty}$ & 102.78 & 100.72 & 104.89 \\
\hline
\end{tabular}

$3 D P$ three-dimensional printing, In natural logarithm, $A U C_{O-T}$ area under the plasma concentration-time curve from time zero to the time of the last measurable concentration, $A U C_{0-\infty}$ area under the plasma concentration-time curve from time zero to infinity, $C_{\max }$ maximum observed plasma concentration

provide the greatest effects on gastrointestinal physiology. As indicated in Table 4, the administration of a high-fat, high-calorie breakfast delayed the median $T_{\max }$ by approximately $3.5 \mathrm{~h}$ and resulted in a decrease of $C_{\max }$. The $C_{\max }$ geometric LS mean of levetiracetam in the fed state was $63.9 \%$ of the peak value of the fasting state but the food intake had no effect on the extent of systemic exposure as expressed by AUC (Table 6). The mean concentration-time curves of levetiracetam 3DP fast-melt formulation under fasting and fed conditions are depicted in Fig. 2.

\subsection{Sex Subgroup Analyses}

The administration of a 1000-mg levetiracetam dose was further evaluated in male and female subgroups (Table 7). Higher concentrations and AUCs were observed with both formulations (3DP fast melt and conventional tablet) in female subjects compared with male subjects. The median
Table 6 Ninety percent confidence intervals of ln-transformed pharmacokinetic parameters between the administration of levetiracetam 3DP fast melt under fed conditions vs. levetiracetam 3DP fast melt under fasting conditions in healthy volunteers

\begin{tabular}{llll}
\hline Parameter & \multirow{2}{*}{ Ratio (fed/fast) } & \multicolumn{2}{l}{$90 \%$ confidence intervals } \\
\cline { 3 - 4 } & & Lower & Upper \\
\hline $\ln C_{\text {max }}$ & 63.85 & 59.79 & 68.19 \\
$\ln A U C_{0-\mathrm{T}}$ & 94.45 & 92.58 & 96.36 \\
$\operatorname{lnAUC}_{0-\infty}$ & 94.93 & 93.00 & 96.89 \\
\hline
\end{tabular}

$3 D P$ three-dimensional printing, $A U C_{O-T}$ area under the plasma concentration-time curve from time zero to the time of the last measurable concentration, $A U C_{0-\infty}$ area under the plasma concentration-time curve from time zero to infinity, $C_{\max }$ maximum observed plasma concentration, In natural logarithm

$T_{\max }$ showed a slight delay: $0.5 \mathrm{~h}(1 \mathrm{vs} .0 .5 \mathrm{~h})$ for the 3DP fast melt (test) in female subjects, but was similar for the conventional tablet (reference) in both subgroups. The apparent elimination half-life was shorter for women when compared with that of male subjects ( $\sim 6.5$ vs. 7.6 and $\sim 6.3$ vs. $7.8 \mathrm{~h}$, respectively) for both formulations.

When sex was added as a fixed effect into the default mixed-effect ANOVA model, significant differences between female and male subjects were detected for all observed ln-transformed PK parameters ( $p$ value $C_{\max }$ $0.0002, \mathrm{AUC}_{0-\mathrm{T}} 0.0004$ and $\left.\mathrm{AUC}_{0-\infty} 0.0015\right)$ for the $3 \mathrm{DP}$ fast melt vs. the conventional tablet under fasting conditions (at $5 \%$ significance level). Separate analysis was done for ranked $T_{\max }$ and significant difference was also detected ( $p=0.0439$ ). Differences remain significant after normalization of the ln-transformed PK parameters considering the individual weight and dose $\left(p\right.$ value $C_{\max }$ $0.0019, \mathrm{AUC}_{0-\mathrm{T}} 0.0002$, and $\mathrm{AUC}_{0-\infty}$ 0.0002).
Fig. 1 Mean levetiracetam plasma concentration-time (+standard deviation) curve profiles (linear scale) after oral administration of a single 1000-mg dose of test formulation and a single 1000-mg dose of reference tablet. 3DP three-dimensional printing

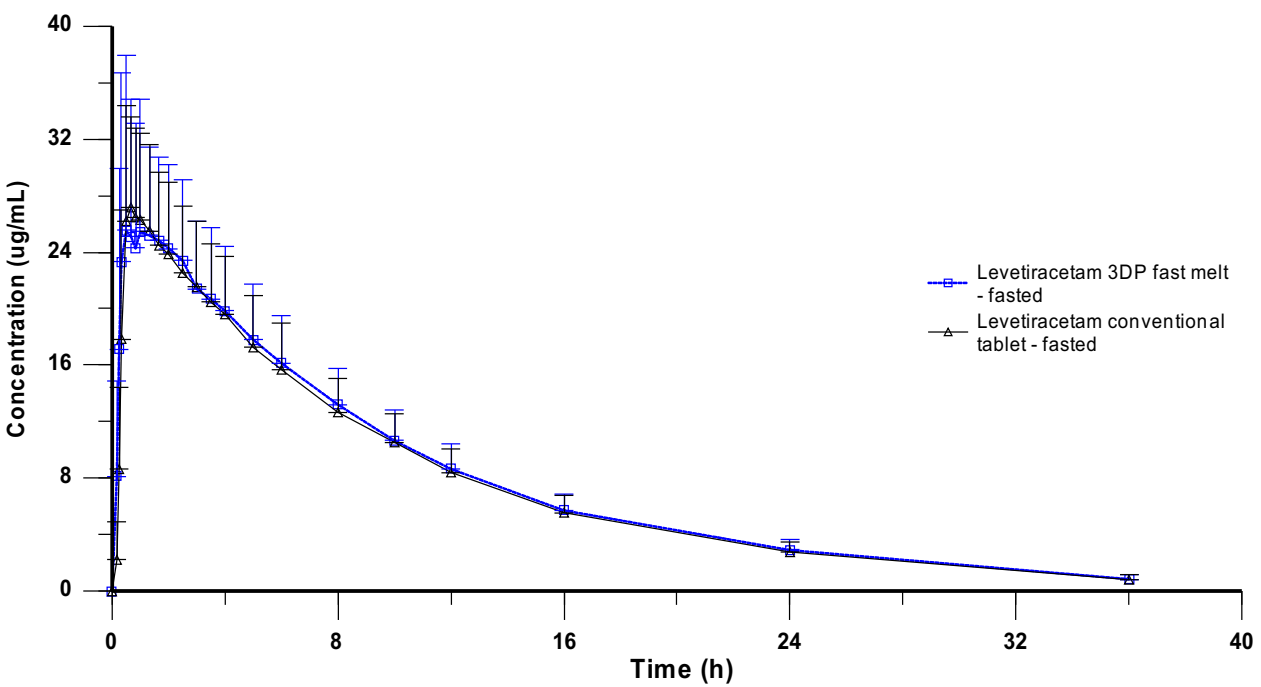


Fig. 2 Mean plasma concentration-time (+standard deviation) curve profiles (linear scale) of levetiracetam 3DP fast melt following the administration of a high-fat, high-calorie breakfast compared with fasting conditions. $3 D P$ three-dimensional printing

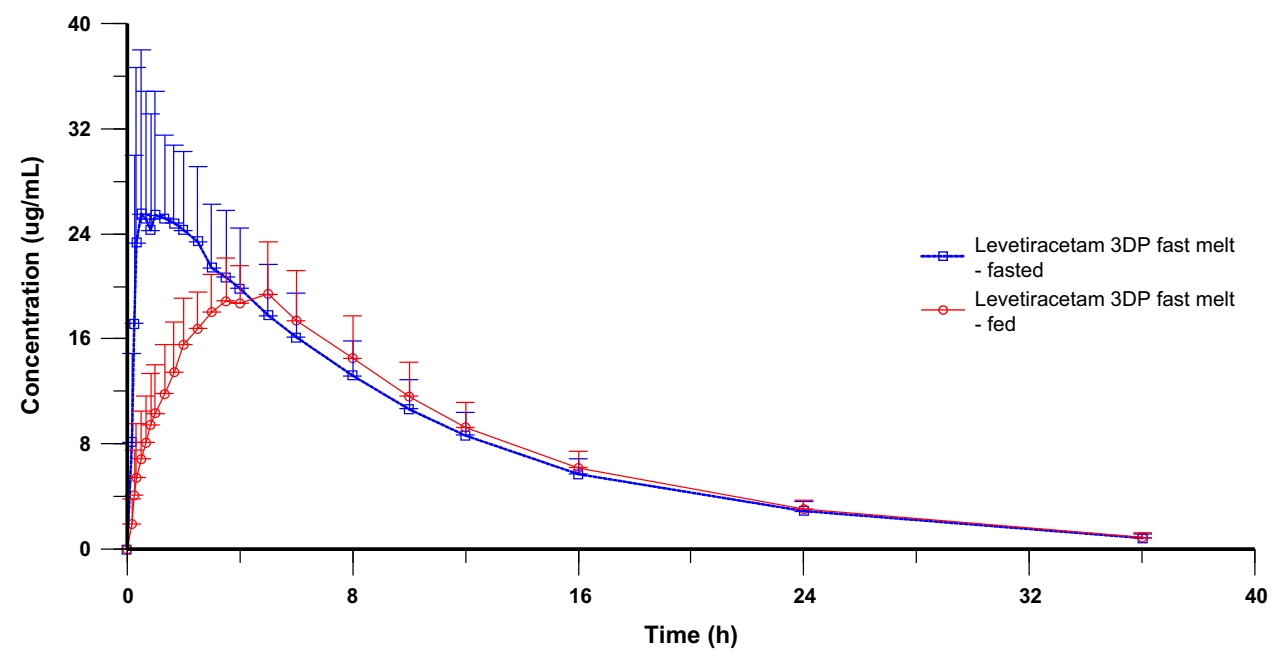

Table 7 Comparison of the pharmacokinetic parameters between female and male subjects for the levetiracetam 3DP fast melt and levetiracetam conventional tablet under fasting conditions

\begin{tabular}{|c|c|c|c|c|c|c|c|c|}
\hline \multirow[t]{3}{*}{ Parameter } & \multicolumn{4}{|c|}{ Female subjects } & \multicolumn{4}{|c|}{ Male subjects } \\
\hline & \multicolumn{2}{|c|}{$\begin{array}{l}\text { Levetiracetam } \\
\text { 3DP fast melt } \\
\text { Fasted } \\
N=14\end{array}$} & \multicolumn{2}{|c|}{$\begin{array}{l}\text { Conventional tablet } \\
\text { Fasted } \\
N=14\end{array}$} & \multicolumn{2}{|c|}{$\begin{array}{l}\text { Levetiracetam } \\
\text { 3DP fast melt } \\
\text { Fasted } \\
N=18\end{array}$} & \multicolumn{2}{|c|}{$\begin{array}{l}\text { Conventional tablet } \\
\text { Fasted } \\
N=18\end{array}$} \\
\hline & Mean $^{a}$ & (c.v.\%) & Mean $^{a}$ & (c.v.\%) & $\operatorname{Mean}^{\mathrm{a}}$ & (c.v.\%) & Mean $^{a}$ & (c.v.\%) \\
\hline$T_{\max }(\mathrm{h})$ & 1.00 & $(68.5)$ & 0.67 & $(75.3)$ & 0.50 & $(62.4)$ & 0.50 & $(57.2)$ \\
\hline$C_{\max }(\mu \mathrm{g} / \mathrm{mL})$ & 38.190 & $(22.4)$ & 34.475 & (13.6) & 29.448 & $(32.5)$ & 27.372 & $(16.7)$ \\
\hline $\operatorname{AUC}_{0-\mathrm{T}}(\mu \mathrm{g} \cdot \mathrm{h} / \mathrm{mL})$ & 323.946 & $(17.0)$ & 304.702 & $(17.0)$ & 252.379 & (13.6) & 251.780 & $(13.9)$ \\
\hline $\mathrm{AUC}_{0-\infty}(\mu \mathrm{g} \cdot \mathrm{h} / \mathrm{mL})$ & 331.870 & $(17.8)$ & 312.097 & $(17.3)$ & 262.639 & (13.9) & 262.679 & $(14.4)$ \\
\hline$T_{\text {half }}(\mathrm{h})$ & 6.47 & $(12.2)$ & 6.34 & $(15.0)$ & 7.641 & (9.6) & 7.77 & $(11.7)$ \\
\hline
\end{tabular}

$T_{\max }$ time to maximum observed plasma concentration, $C_{\max }$ maximum observed plasma concentration, $A U C_{0-T}$ area under the plasma concentration-time curve from time zero to the time of the last measurable concentration, $A U C_{0-\infty}$ area under the plasma concentration-time curve from time zero to infinity, $T_{\text {half }}$ terminal elimination half-life, c.v. coefficient of variation, $3 D P$ three-dimensional printing

${ }^{a}$ Median for $T_{\max }$

\subsection{Water Intake}

No significant trend or correlation was observed between the amount of water ingested with the 3DP fast melt vs. the measured levetiracetam plasma concentrations nor the AUC $\left(C_{\max } p\right.$ value for the Pearson correlation coefficient 0.5100 and 0.7602 , fasting and fed, respectively; $\mathrm{AUC}_{0-\mathrm{T}}$ $p$ value 0.6229 and 0.7058 , fasting and fed, respectively) (Fig. 3).

\subsection{Safety}

Single 1000-mg oral dose of the levetiracetam 3DP fast melt (test product) was generally well tolerated when administered under fasting and fed states. No medications were taken between the screening visit and the first dosing. In addition, no AEs required the use of medications following the first dosing until the end of the study. The incidence of drug-related AEs reported was notably lower for levetiracetam (test) administered under fed conditions $(34 \%)$ than for levetiracetam (test) and the reference levetiracetam products administered under fasting conditions (each $56 \%$ ). The majority of AEs reported during the study were of mild $(n=79)$ or moderate $(n=15)$ severity (Table 8$)$. No severe AEs were observed during the study. None of the AEs reported after the administration of levetiracetam was serious. No adverse events required the use of medications following the first dosing.

The most frequently reported drug-related AEs during the study were of nervous system origin. The most common adverse events included somnolence, dizziness, and fatigue.

No deaths or serious AEs were reported in the present study. No subject was withdrawn from the study for safety reasons. 
Fig. 3 Effect of water intake on pharmacokinetic parameters. a Under fasting conditions. b Under fed conditions. $C_{\max }$ maximum observed plasma concentration, $A U C_{O-T}$ area under the plasma concentrationtime curve from time zero to the time of the last measurable concentration, extotal total volume of water consumed by subject
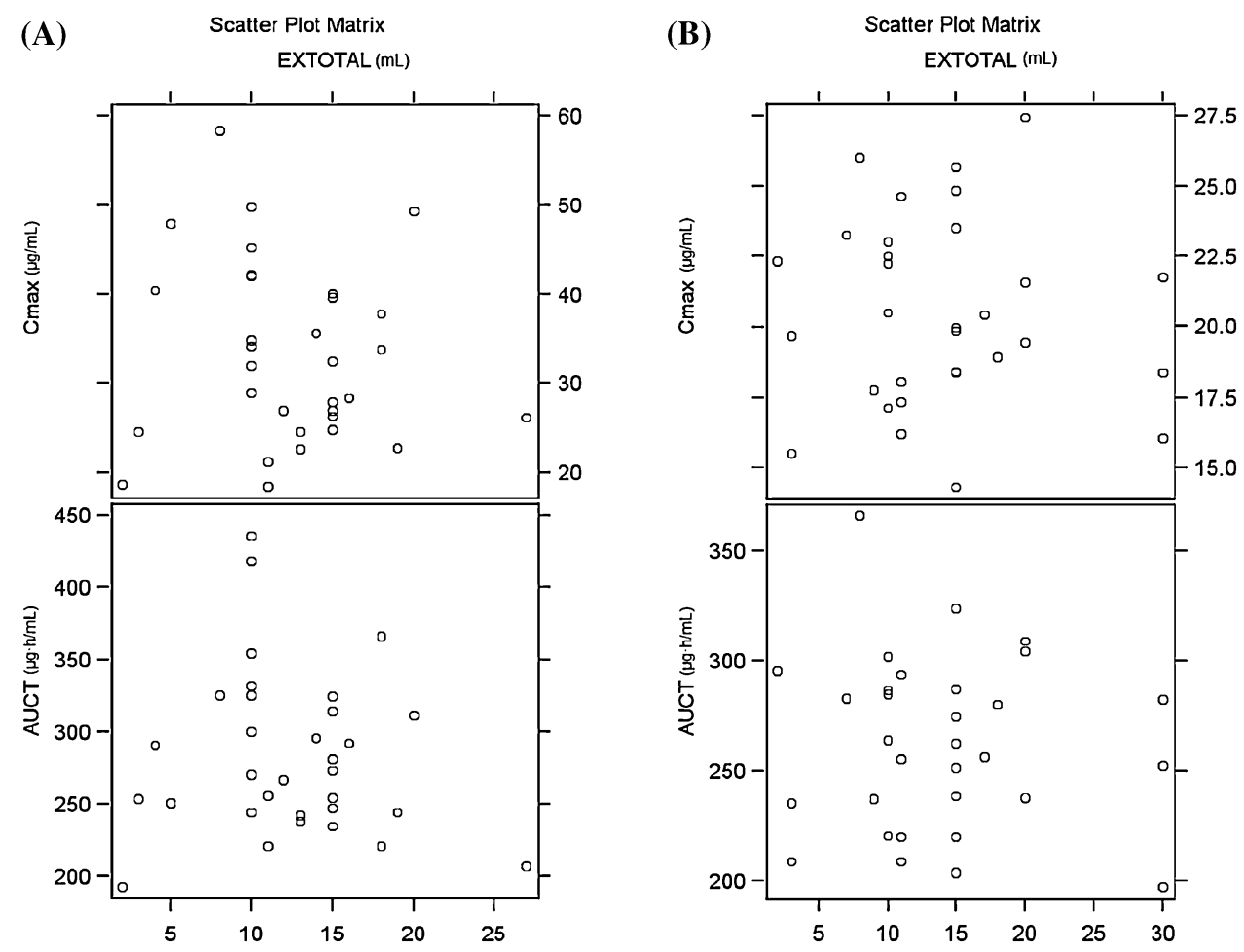

Table 8 Number of adverse events per treatment, classified by severity and causality

\begin{tabular}{|c|c|c|c|c|c|}
\hline \multirow[t]{3}{*}{ Treatments } & \multicolumn{5}{|c|}{ Adverse events $(n)$} \\
\hline & \multicolumn{3}{|c|}{ Severity } & \multicolumn{2}{|l|}{ Causality } \\
\hline & Mild & Moderate & Severe & Reasonable possibility & No reasonable possibility \\
\hline Treatment-1 (test fast) & 32 & 7 & 0 & 33 & 6 \\
\hline Treatment-2 (reference fast) & 31 & 4 & 0 & 28 & 7 \\
\hline Treatment-3 (test fed) & 16 & 4 & 0 & 13 & 7 \\
\hline Total no. of adverse events & 79 & 15 & 0 & 74 & 20 \\
\hline
\end{tabular}

\section{Discussion}

Powder-liquid 3DP technology is being used to produce high-dose solid oral products that can be offered in a 'fast-melt' or rapidly disintegrating formulation, to aid in patient compliance and ensure ease of dosing and swallowing. Fast melts manufactured by the 3DP process are essentially wet-bound, uncompressed, highly porous formulations with a shape similar to conventional tablets. However, unlike conventional tablets, 3DP formulated fast melts rapidly disintegrate within seconds in the mouth, when exposed to a small volume of liquid. Taking 3DP formulated fast melts with a sip of liquid not only facilitates rapid dispersion in the mouth but also aids in the subsequent swallowing of the dispersed medication.
Unlike liquid solutions or suspensions, this mode of administration does not require measurement or preparation outside the body, and ensures the entire dose may be consumed without the question of residue on a measuring device (e.g., cup or oral syringe).

\subsection{Pharmacokinetics}

Aprecia Pharmaceuticals Company developed a novel 1000-mg levetiracetam 3DP fast-melt formulation that was designed to provide plasma concentration and systemic exposure comparable to the reference product after administration of a single dose in normal healthy volunteers. Moreover, the effect of food consumption on the PK profile of this novel formulation was evaluated. 
As noted above, the 3DP fast-melt formulation of levetiracetam is designed to disperse rapidly in the mouth when taken with a small amount of liquid. The actual measurement of in situ disintegration times in the study subjects showed that the tablets rapidly dispersed in the mouth when taken with a sip of water (mean time from sip to swallowing of $11 \mathrm{~s}$ ), which is consistent with the in vitro disintegration measurements (e.g., dispersion occurs on average in 5-6 s for the 1000-mg formulation) [10]. The amount of water used for dosing the product (test product administered with a sip consisting of 2-30 mL; reference product administered with $240 \mathrm{~mL}$ water) did not affect the absorption of the drug and overall PK parameters were comparable. Moreover, a variable amount of water taken from the sip did not show any correlation with AUC or $C_{\max }$ for the 3DP fast-melt product.

As described before, the three-way crossover design was found suitable to meet the objectives of the study. The sample size was based on previous in-house data at Algorithme Pharma to meet an expected test to reference ratio of geometric LSmeans within $92.5 \%$ and $107.5 \%$ and a power of at least $80 \%$. Sufficient subjects were enrolled to account for the possibility of drop-outs, variations around the estimated intra-subject coefficient of variation, and to conclude in favor of the hypothesis of relative bioavailability with sufficient statistical power.

The results show that ratio of geometric LSmeans and corresponding $90 \% \mathrm{CI}$ for the $C_{\max }, \mathrm{AUC}_{0-\mathrm{T}}$, and $\mathrm{AUC}_{0-\infty}$ were all within the FDA BE acceptance range of 80.00-125.00\%, indicating that the 3DP levetiracetam fast melt, as administered with a sip of liquid, is equivalent in rate and extent of absorption to the conventional levetiracetam tablet administered normally under fasting conditions.

A subset analysis by sex indicated that the bioavailability of levetiracetam was significantly higher in female subjects compared with male subjects under fasting conditions for both levetiracetam 3DP fast melt and conventional tablet. Normalization by weight and dose could not explain the differences. Coupez et al. also reported larger $C_{\max }$ and AUCs in female subjects and a delay in $T_{\max }$ with the administration of an oral levetiracetam solution [2].

Administration of a drug product with food may change the bioavailability by affecting either the drug substance or the drug product [11]. The effect of food on the levetiracetam 3DP fast-melt formulation was evaluated using a high-fat, high-calorie breakfast. The $C_{\max }$ geometric LS mean of levetiracetam in the fed state was $63.9 \%$ of the peak value of the fasting state. Additionally, the median time to peak concentration was significantly delayed under fed conditions by approximately $3.5 \mathrm{~h}$. However, the food intake had no effect on the extent of the systemic exposure. Additionally, the mean apparent terminal elimination half- life $\left(T_{\text {half }}\right)$ was similar in both conditions. Thus, food significantly decreased peak values and delayed peak times of the levetiracetam 1000-mg 3DP fast-melt formulation but not the extent of absorption. These observations are consistent with the PKs of the conventional levetiracetam tablets in the fed state where a decrease $C_{\max }$ of $20 \%$ with a delay of $4 \mathrm{~h}$ are reported [1]. Other studies also report a delay in the absorption with a small decrease in the exposure by $8-10 \%$ [12], or in contrast describe the absence of drug-food interactions [13]. None of the reported effects were deemed of clinical significance.

\subsection{Safety}

The new 3DP fast-melt formulation of levetiracetam was found to be safe and well tolerated when administered to healthy volunteers. The most commonly experienced adverse events included somnolence, dizziness, and fatigue. The safety profile observed in this study corresponds to the one presented in the product's monograph where the most commonly reported AEs included somnolence, asthenia, infection, and dizziness [1]. Furthermore, behavioral/psychiatric symptoms such as hallucinations, paranoid reaction, psychosis, and psychotic depression; as well as coordination difficulties such as ataxia, abnormal gait, and incoordination were shown to be reported after the administration of levetiracetam. However, no abnormal behavioral and neurological examinations were recorded during this study. Serious hypersensitivity reactions with dermatological involvement have been reported in association with levetiracetam use [1]. In the present study, no changes on the oral mucosa were noted for any subject from the pre- to the post-dose evaluation. Minor hematological abnormalities were also reported in controlled clinical trials in patients treated with levetiracetam for total mean red blood cell count, mean hemoglobin, and mean hematocrit [14]. One subject presented ecchymosis on the thighs; however, her hematology tests were considered not clinically significant. On-study vital signs and ECGs were of no clinical significance.

Interestingly, the number of AEs related to the levetiracetam 3DP fast melt was lower under fed conditions; this could be related to the delayed and/or the lower peak plasma values after the intake of food.

\section{Conclusions}

No clinically significant effects on vital signs, ECGs, neurological examinations, or the oral mucosa were noted and no serious AEs were reported during the study. A single 1000-mg oral dose of the levetiracetam 3DP fastmelt product was generally safe and well tolerated by the 
subjects included in this study. The safety profile of the levetiracetam 3DP fast melt product was similar under both fed and fasting conditions, and similar to the profile presented in the conventional or reference product's monograph. Overall, most subjects agreed that the mouth feel of the 3DP fast-melt product was acceptable and easy to take and swallow. In addition, the majority indicated the 3DP fast-melt product disintegrated or melted quickly in their mouth prior to swallowing, when taken with a small sip of water.

The present study concludes that the levetiracetam 3DP fast melt is equivalent in rate and extent of absorption to the conventional immediate-release tablet when given in the fasted state. The rapid oral disintegration for the 3DP fast melt did not affect the PK profile of levetiracetam even though a delay and a lower peak in the absorption were observed under fed conditions. These effects are unlikely to be of clinical significance with long-term administration, and may help to reduce the AEs and facilitate compliance.

Acknowledgments The authors wish to thank Mr. Doug Plessinger and Dr. Nemichand Jain from Aprecia Pharmaceuticals Company for the helpful discussion on the 3DP manufacturing process and for their review of the present manuscript.

\section{Compliance with Ethical Standards}

This study was conducted in compliance with the study protocol, the ethical principles that have their origins in the Declaration of Helsinki, and the ICH Guideline E6 for GCP. Approval was obtained from an independent ethics committee and from Health Canada. Written informed consent was obtained from participants prior to study commencement.

This study was funded by Aprecia Pharmaceuticals Company, and the study was conducted by the Contract Research Organisation Algorithme Pharma Inc. The sponsor did not participate in the execution of the study or in the analysis of the data. Sophie Boudriau, Cecilia Hanzel, Julie Massicotte, Laura Sayegh, Jing Wang, and Marc Lefebvre are full-time employees and/or stockholders of Algorithme Pharma Inc.

Open Access This article is distributed under the terms of the Creative Commons Attribution-NonCommercial 4.0 International License (http://creativecommons.org/licenses/by-nc/4.0/), which permits any noncommercial use, distribution, and reproduction in any medium, provided you give appropriate credit to the original author(s) and the source, provide a link to the Creative Commons license, and indicate if changes were made.

\section{References}

1. Keppra ${ }^{\circledR}$ [Product Monograph] (May 13, 2009). Drugs approvals and databases, FDA. Available from: http://www.accessdata.fda. gov/drugsatfda_docs/label/2009/021035s078s080,021505s021s024 lbl.pdf. Accessed 22 Dec 2015.

2. Coupez R, Straetemans R, Sehgal G, Stockis A, Lu ZS et al. Levetiracetam: relative bioavailability and bioequivalence of a $10 \%$ oral solution $(750 \mathrm{mg}$ ) and $750 \mathrm{mg}$ tablets. J Clin Pharmacol. 2003;43:1370-6.

3. Orange book: approved drug products with therapeutic equivalence evaluations. U.S. Food and Drug Administration (FDA). Available from: http://www.accessdata.fda.gov/scripts/cder/ob/ docs/queryai.cfm. Levetiracetam. Accessed 22 Dec 2015.

4. Gold DT, Safi W, Trinh H. Patient preference and adherence: comparative US studies between two bisphosphonates, weekly risedronate and monthly ibandronate. Curr Med Res Opin. 2006;22:2383-91.

5. Masand PS, Narasimhan M. Improving adherence to antipsychotic pharmacotherapy. Curr Clin Pharmacol. 2006;1:47-56.

6. Schindler JS, Kelly JH. Swallowing disorders in the elderly. Laryngoscope. 2002;112:589-602.

7. Regan J, Sowman R, Walsh I. Prevalence of dysphagia in acute and community mental health settings. Dysphagia. 2006;21:95-101.

8. Singh J, Garg R, Gupta GD. Enhancement of solubility of lamotrigine by solid dispersion and development of orally disintegrating tablets using 32 full factorial design. J Pharm (Cairo). 2015;5:828453.

9. Sajatovic M, Thompson TR, Nanry K, Edwards S, Manjunath R. Prospective, open-label trial measuring satisfaction and convenience of two formulations of lamotrigine in subjects with mood disorders. Patient Prefer Adherence. 2013;7:411-7.

10. Aprecia Pharmaceuticals. Data on file. Sep 2015.

11. Guidance for Industry. Food effect bioavailability and fed studies. Food and Drug Administration Center for Drug Evaluation and Research (CDER). U.S. Food and Drug Administration (FDA). Dec 2002. Available from http://www.fda.gov/ucm/groups/fda gov-public/@fdagov-afda-gen/documents/document/ucm126833. pdf

12. Zhao Q, Jiang J, Li X, Lu Z, Hu P. Single-dose pharmacokinetics of levetiracetam in healthy Chinese male subjects. Br J Clin Pharmacol. 2006;63:614-7.

13. Radtke RA. Pharmacokinetics of levetiracetam. Epilepsia. 2001;42(Suppl 4):24-7.

14. Stefan H, Feuerstein TJ. Novel anticonvulsant drugs. Pharmacol Ther. 2007;113:165-83. 